\title{
Transition density and pressure in hot neutron stars
}

\author{
Jun $\mathrm{Xu},{ }^{1}$ Lie-Wen Chen, ${ }^{2,3}$ Che Ming Ko, ${ }^{4}$ and Bao-An $\mathrm{Li}^{5}$ \\ ${ }^{1}$ Cyclotron Institute, Texas A\&M University, College Station, Texas 77843-3366, USA \\ ${ }^{2}$ Department of Physics, Shanghai Jiao Tong University, Shanghai 200240, China \\ ${ }^{3}$ Center of Theoretical Nuclear Physics, National Laboratory of Heavy Ion Accelerator, Lanzhou 730000, China \\ ${ }^{4}$ Cyclotron Institute and Department of Physics and Astronomy, Texas A\&M University, College Station, Texas 77843-3366, USA \\ ${ }^{5}$ Department of Physics and Astronomy, Texas A\&M University-Commerce, Commerce, Texas 75429-3011, USA
}

(Received 22 March 2010; revised manuscript received 21 April 2010; published 24 May 2010)

\begin{abstract}
Using the momentum-dependent effective interaction (MDI) for nucleons, we have studied the transition density and pressure at the boundary between the inner crust and the liquid core of hot neutron stars. We find that their values are larger in neutrino-trapped neutron stars than in neutrino-free neutron stars. Furthermore, both are found to decrease with increasing temperature of a neutron star as well as increasing slope parameter of the nuclear symmetry energy, except that the transition pressure in neutrino-trapped neutron stars for the case of small symmetry energy slope parameter first increases and then decreases with increasing temperature. We have also studied the effect of the nuclear symmetry energy on the critical temperature above which the inner crust in a hot neutron star disappears and found that with increasing value of the symmetry energy slope parameter, the critical temperature decreases slightly in neutrino-trapped neutron stars but first decreases and then increases in neutrino-free neutron stars.
\end{abstract}

DOI: 10.1103/PhysRevC.81.055805

PACS number(s): 26.60.-c, 21.30.Fe, 21.65.-f, 97.60.Jd

\section{INTRODUCTION}

Studying the properties of neutron stars allows us to test our knowledge on the properties of nuclear matter under extreme conditions. Theoretical studies have shown that a neutron star is expected to have a liquid core surrounded by an inner crust [1], which extends outward to the neutron drip-out region. While the neutron drip-out density $\rho_{\text {out }}$ has been relatively well determined [2], the transition density $\rho_{t}$ at the inner edge of the crust is still quite uncertain because of our limited knowledge of the nuclear equation of state (EOS), especially the density dependence of the symmetry energy $\left[E_{\mathrm{sym}}(\rho)\right]$ of neutron-rich nuclear matter [3,4]. Recently, significant progress has been made in constraining the EOS of neutron-rich nuclear matter using terrestrial laboratory experiments (see Ref. [5] for a recent review). In particular, from analyses of experimental data on neutron skin thickness, isobaric analog states, pygmy dipole resonances, and giant dipole resonances in nuclei as well as on isospin diffusion, isoscaling, and neutronproton double ratio in intermediate-energy nuclear reactions, significant constraints on $E_{\text {sym }}(\rho)$ have been obtained for the same subsaturation density region as expected in the inner edge of neutron star crusts. The extracted slope parameter $L=3 \rho_{0}\left[\partial E_{\text {sym }}(\rho) / \partial \rho\right]_{\rho=\rho_{0}}$ of the nuclear symmetry energy from these studies has values in the range $30 \mathrm{MeV}<L<$ $80 \mathrm{MeV}$ [6]. With the momentum-dependent interaction (MDI) together with the value $L=86 \pm 25 \mathrm{MeV}$ constrained from an analysis of the isospin-diffusion data [7-10] in heavy-ion collisions using the isospin-dependent Boltzmann-UehlingUhlenbeck (IBUU) transport model with the MDI [11], the density and pressure at the inner edge of the crust of cold neutron stars were studied by considering the boundary of the instability region or the spinodal boundary between the liquid core and the inner crust of a cold neutron star in both the thermodynamical approach $[4,12]$ and the dynamical approach [13-17]. This leads to the constraints $0.040 \mathrm{fm}^{-3}<$ $\rho_{t}<0.065 \mathrm{fm}^{-3}$ and $0.01 \mathrm{MeV} / \mathrm{fm}^{3}<P_{t}<0.26 \mathrm{MeV} / \mathrm{fm}^{3}$, respectively, for the transition density and pressure. Together with the crustal fraction of the total moment inertia of the Vela pulsar extracted from its glitches [18], a tighter constraint on the mass-radius relation of cold neutron stars was obtained [19].

Because of the initial high temperature and appreciable proton fraction in a newly formed neutron star immediately after gravitational collapse of a massive star [20-22], neutrinos are abundantly produced from the Urca process in its inner core. Although high-energy neutrinos can be trapped at densities as low as $10^{12} \mathrm{~g} / \mathrm{cm}^{3}$ [23], the star cools by neutrino emissions. As neutrinos are emitted from this so-called protoneutron star, which has an initial temperature of $\sim 10^{11} \mathrm{~K}$ (about $10 \mathrm{MeV}$ ) [24,25], its temperature drops to $\sim 10^{10} \mathrm{~K}$ (about $1 \mathrm{MeV}$ ) and even lower. Afterward, the neutron star becomes transparent to neutrinos as their mean free path increases with decreasing energy, and the cooling of the neutron star continues to be dominated by neutrino emission for a long time. It is thus of interest to study the transition density and pressure in newly born hot neutron stars, as this would help to understand the cooling mechanism and structural evolution of neutron stars. In this article, we extend the study of Ref. [19] to finite temperature and study the dependence of the transition density and pressure of hot neutron stars on the nuclear symmetry energy, particularly its slope at nuclear saturation density.

This article is organized as follows. We first review in Sec. II the MDI for nucleons, in Sec. III the properties of hot neutron star matter, and in Sec. IV the dynamical approach for locating the inner edge of the crust of a hot neutron star. We then show in Sec. V the results and conclude with a summary in Sec. VI. 


\section{THE MDI}

The MDI is an effective nuclear interaction with its density and momentum dependence constrained from the phenomenological finite-range Gogny interaction [11]. In the mean-field approximation, the potential energy density of a nuclear matter of density $\rho$ and isospin asymmetry $\delta=\left(\rho_{n}-\rho_{p}\right) / \rho$, with $\rho_{n}$ and $\rho_{p}$ being, respectively, the neutron and proton densities, can be expressed as $[8,11]$

$$
\begin{aligned}
V(\rho, \delta)= & \frac{A_{u}(x) \rho_{n} \rho_{p}}{\rho_{0}}+\frac{A_{l}(x)}{2 \rho_{0}}\left(\rho_{n}^{2}+\rho_{p}^{2}\right) \\
& +\frac{B}{\sigma+1} \frac{\rho^{\sigma+1}}{\rho_{0}^{\sigma}}\left(1-x \delta^{2}\right) \\
& +\frac{1}{\rho_{0}} \sum_{\tau, \tau^{\prime}} C_{\tau, \tau^{\prime}} \iint d^{3} p d^{3} p^{\prime} \frac{f_{\tau}(\vec{r}, \vec{p}) f_{\tau^{\prime}}\left(\vec{r}^{\prime}, \vec{p}^{\prime}\right)}{1+\left(\vec{p}-\vec{p}^{\prime}\right)^{2} / \Lambda^{2}}
\end{aligned}
$$

In the preceding equation, $\tau\left(\tau^{\prime}\right)$ is the nucleon isospin taken to be $1 / 2$ for neutron and $-1 / 2$ for proton; $f_{\tau}(\vec{r}, \vec{p})=$ $\left(2 / h^{3}\right)\left\{\exp \left[\left(p^{2} / 2 m+U_{\tau}-\mu_{\tau}\right) / T\right]+1\right\}^{-1}$ is the nucleon phase-space distribution function in a thermally equilibrated nuclear matter with $m=939 \mathrm{MeV}$ being the nucleon mass, $\mu_{\tau}$ being the chemical potential of nucleon of isospin $\tau, T$ being the temperature, and $U_{\tau}$ being the nucleon mean-field potential to be introduced in what follows; and $\rho_{0}=0.16 \mathrm{fm}^{-3}$ is the saturation density of normal nuclear matter. Values of the parameters $A_{u}(x), A_{l}(x), B, \sigma, \Lambda, C_{l}=C_{\tau, \tau}$, and $C_{u}=C_{\tau,-\tau}$ can be found in Refs. [8,11]. For symmetric nuclear matter, this interaction gives a binding energy of $-16 \mathrm{MeV}$ per nucleon and an incompressibility $K_{0}$ of $212 \mathrm{MeV}$ at saturation density.

Taking the derivative of Eq. (1) with respect to the proton or neutron density leads to the following single-particle potential for a nucleon of isospin $\tau$ :

$$
\begin{aligned}
U(\rho, \delta, \vec{p}, \tau)= & A_{u}(x) \frac{\rho_{-\tau}}{\rho_{0}}+A_{l}(x) \frac{\rho_{\tau}}{\rho_{0}}+B\left(\frac{\rho}{\rho_{0}}\right)^{\sigma}\left(1-x \delta^{2}\right) \\
& -8 \tau x \frac{B}{\sigma+1} \frac{\rho^{\sigma-1}}{\rho_{0}^{\sigma}} \delta \rho_{-\tau} \\
& +\frac{2 C_{\tau, \tau}}{\rho_{0}} \int d^{3} p^{\prime} \frac{f_{\tau}\left(\vec{r}, \vec{p}^{\prime}\right)}{1+\left(\vec{p}-\vec{p}^{\prime}\right)^{2} / \Lambda^{2}} \\
& +\frac{2 C_{\tau,-\tau}}{\rho_{0}} \int d^{3} p^{\prime} \frac{f_{-\tau}\left(\vec{r}, \vec{p}^{\prime}\right)}{1+\left(\vec{p}-\vec{p}^{\prime}\right)^{2} / \Lambda^{2}}
\end{aligned}
$$

which is seen to depend on the momentum $\vec{p}$ of the nucleon. Although the properties of cold nuclear matter can be essentially determined analytically using Eqs. (1) and (2), these equations need to be solved numerically and self-consistently by the iteration method [26] to obtain the thermodynamical quantities of hot nuclear matter.

In cold nuclear matter, the symmetry energy from the MDI is given by

$$
\begin{aligned}
E_{\mathrm{sym}}(\rho) & =\frac{1}{2}\left(\frac{\partial^{2} E}{\partial \delta^{2}}\right)_{\delta=0} \\
& =\frac{8 \pi}{9 m h^{3} \rho} p_{f}^{5}+\frac{\rho}{4 \rho_{0}}[-24.59+4 B x /(\sigma+1)]
\end{aligned}
$$

$$
\begin{aligned}
& -\frac{B x}{\sigma+1}\left(\frac{\rho}{\rho_{0}}\right)^{\sigma}+\frac{C_{l}}{9 \rho_{0} \rho}\left(\frac{4 \pi}{h^{3}}\right)^{2} \Lambda^{2} \\
& \times\left[4 p_{f}^{4}-\Lambda^{2} p_{f}^{2} \ln \left(\frac{4 p_{f}^{2}+\Lambda^{2}}{\Lambda^{2}}\right)\right] \\
& +\frac{C_{u}}{9 \rho_{0} \rho}\left(\frac{4 \pi}{h^{3}}\right)^{2} \Lambda^{2} \\
& \times\left[4 p_{f}^{4}-p_{f}^{2}\left(4 p_{f}^{2}+\Lambda^{2}\right) \ln \left(\frac{4 p_{f}^{2}+\Lambda^{2}}{\Lambda^{2}}\right)\right],
\end{aligned}
$$

where $p_{f}=\hbar\left(3 \pi^{2} \rho / 2\right)^{1 / 3}$ is the nucleon Fermi momentum in symmetric nuclear matter. The first term in Eq. (3) is the contribution from the kinetic part, while other terms are from the potential part. The symmetry energy is fixed to be $30.5 \mathrm{MeV}$ at normal nuclear density, and the parameter $x$ is used to model the density dependence of the symmetry energy away from the saturation density without changing the properties of symmetric nuclear matter. The resulting slope parameter of the symmetry energy has values of about 15,60 , and $106 \mathrm{MeV}$ for $x=1,0$, and -1 , respectively.

The density dependence of the symmetry energy from the MDI is shown in Fig. 1(a) for $x=1, x=0$, and $x=-1$. With $x=1(x=-1)$ the symmetry energy is larger (smaller) at subsaturation densities but smaller (larger) at suprasaturation densities, and it is called a "soft" ("stiff") symmetry energy. For $x=0$, the value of the symmetry energy lies between those of $x=1$ and $x=-1$. In Figs. 1(b) and 1(c), the momentum dependence of the nucleon single-particle potential $U_{0}$ in symmetric nuclear matter at half saturation density is displayed. Also shown in the figure is the symmetry potential, defined as $U_{\text {sym }}=\left(U_{n}-U_{p}\right) / 2 \delta$ and calculated at $\delta=0.2$ and the same density. It is seen that $U_{0}$ has negative values at low momenta but increases with increasing momentum and saturates at high momenta. The symmetry potential $U_{\text {sym }}$, which measures the difference between the neutron and proton single-particle potentials in an asymmetric nuclear

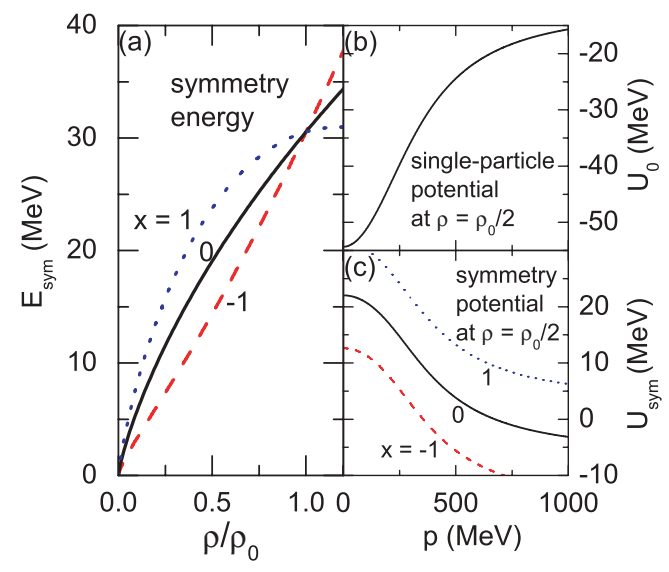

FIG. 1. (Color online) (a) Density dependence of symmetry energies, (b) single-particle potentials in symmetric nuclear matter, and (c) symmetry potentials in cold nuclear matter at $\rho_{0} / 2$ for the MDI. 
matter, decreases with increasing momentum and is larger for $x=1$ than for $x=0$ and $x=-1$, which is consistent with the behavior of the symmetry energy at subsaturation densities.

\section{HOT NEUTRON STAR MATTER}

For a newly born neutron star, its cooling is dominated by the emission of neutrinos, which are produced through the Urca process, leading to the following charge neutrality and $\beta$-stable conditions:

$$
\begin{aligned}
\rho_{p} & =\rho_{e}, \\
\mu_{p}+\mu_{e} & =\mu_{n}+\mu_{v_{e}} .
\end{aligned}
$$

The production of muons is negligible for the density and temperature present in the hot neutron star crust. Neutrino trapping in the early stage of a supernova has been extensively studied in the literature [23], and it was found that the fraction of leptons

$$
Y_{l}=\frac{\rho_{e}+\rho_{v_{e}}}{\rho}
$$

is about $0.35 \sim 0.4$ at the onset of trapping [21,24] and decreases as neutrinos leave the star. In the present study, we consider the two extreme cases of neutrino-trapped and neutrino-free hot neutron star matters. In the former case, we choose $Y_{l}$ to be 0.4 as an example and consider the typical temperature of 5 or $10 \mathrm{MeV}$. In the case of neutrino-free hot neutron star matter, we set the neutrino chemical potential to be 0 and the temperature to be 0 or $1 \mathrm{MeV}$, corresponding to the later stage of a neutron star's evolution. The abundance of each species in both cases can be calculated from the preceding equations together with the baryon number conservation condition $\rho=\rho_{n}+\rho_{p}$.

Assuming that both electrons and neutrinos are massless, we determine the proton fraction $x_{p}=(1-\delta) / 2$ in both the neutrino-trapped matter and the neutrino-free matter as a function of baryon density $\rho$, and the results are shown in Fig. 2. It is seen that the neutrino-free matter is much more neutron-rich than the neutrino-trapped matter. The critical proton fraction $11 \% \sim 15 \%$ for the direct Urca process [27,28] is smaller than the proton fraction in the neutrino-trapped matter but larger than the proton fraction in the neutrino-free matter. The proton fraction increases with increasing density in all cases and slightly increases with increasing temperature for a fixed density, especially at low densities. These can be understood from the increase of the symmetry free energy with increasing temperature and density [26], which makes the neutron star matter more symmetric at higher temperatures and densities. Furthermore, the stiff symmetry energy $(x=-1)$ makes the system more neutron-rich at subsaturation densities, as expected.

The total pressure $P$ in a hot neutron star matter can be written as

$$
P=P_{b}+P_{l},
$$

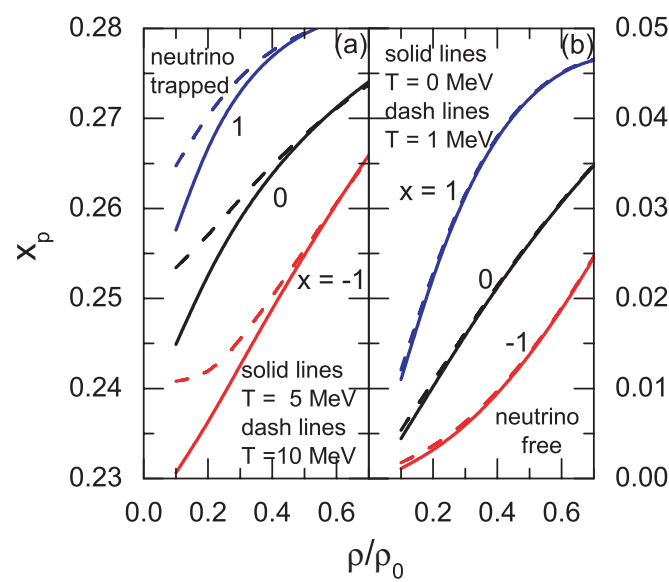

FIG. 2. (Color online) The proton fraction $x_{p}$ as a function of baryon density $\rho$ from the MDI for both neutrino-trapped matter (a) and neutrino-free matter (b) with different $x$ parameters and at different temperatures. Note that different scales for $x_{p}$ are used for the neutrino-free and the neutrino-trapped matter.

where the baryon contribution $P_{b}(\rho, T, \delta)$ is calculated from the thermodynamic relation

$$
\begin{aligned}
P_{b}(\rho, T, \delta)= & {\left[T \sum_{\tau} s_{\tau}(\rho, T, \delta)-V(\rho, T, \delta)\right.} \\
& \left.-V_{\text {kin }}(\rho, T, \delta)\right]+\sum_{\tau} \mu_{\tau} \rho_{\tau} .
\end{aligned}
$$

In the preceding equation, $V(\rho, T, \delta)$ and $V_{\text {kin }}(\rho, T, \delta)$ are, respectively, the potential and kinetic contributions to the total energy density, with the latter given by

$$
V_{\mathrm{kin}}(\rho, T, \delta)=\sum_{\tau} \int d^{3} p \frac{p^{2}}{2 m} f_{\tau}(\vec{r}, \vec{p}),
$$

and $s_{\tau}(\rho, T, \delta)$ is the entropy density, which is given by

$s_{\tau}(\rho, T, \delta)=-\frac{8 \pi}{h^{3}} \int_{0}^{\infty} p^{2}\left[n_{\tau} \ln n_{\tau}+\left(1-n_{\tau}\right) \ln \left(1-n_{\tau}\right)\right] d p$,

with the particle occupation number

$$
n_{\tau}=\frac{1}{\exp \left[\left(p^{2} / 2 m+U_{\tau}-\mu_{\tau}\right) / T\right]+1} .
$$

The preceding formula can also be used for the calculation of $P_{l}$ by using $l\left(l=e, v_{e}\right)$ instead of $\tau$, and leptons are treated as noninteracting ultrarelativistic particles.

\section{LOCATING THE INNER EDGE OF THE NEUTRON STAR CRUST}

We briefly review in this section the dynamical approach that will be used to locate the inner edge of the neutron-star crust [1] and discuss its application to the case of finite temperature. We neglect muons in the neutron star crust as their number is small compared to that of electrons at low densities and temperatures. 
In the dynamical approach, the stability condition for a homogeneous neutron-star matter against small periodic density perturbations can be well approximated by [13-17]

$$
V_{\mathrm{dyn}}(k)=V_{0}+\beta k^{2}+\frac{4 \pi e^{2}}{k^{2}+k_{T F}^{2}}>0,
$$

where $k$ is the wave vector of the spatially periodic density perturbations and

$$
\begin{aligned}
V_{0} & =\frac{\partial \mu_{p}}{\partial \rho_{p}}-\frac{\left(\partial \mu_{n} / \partial \rho_{p}\right)^{2}}{\partial \mu_{n} / \partial \rho_{n}}, \quad k_{T F}^{2}=\frac{4 \pi e^{2}}{\partial \mu_{e} / \partial \rho_{e}}, \\
\beta & =D_{p p}+2 D_{n p} \zeta+D_{n n} \zeta^{2}, \quad \zeta=-\frac{\partial \mu_{n} / \partial \rho_{p}}{\partial \mu_{n} / \partial \rho_{n}} .
\end{aligned}
$$

The three terms in Eq. (12) represent, respectively, contributions from the bulk nuclear matter, the density-gradient terms, and the Coulomb interaction. The empirical values for the coefficients of density-gradient terms are $D_{p p}=D_{n n}=$ $D_{n p}=132 \mathrm{MeV} \mathrm{fm}^{5}[17,19]$. At $k_{\min }=\left[\left(4 \pi e^{2} / \beta\right)^{1 / 2}-\right.$ $\left.k_{T F}^{2}\right]^{1 / 2}, V_{\mathrm{dyn}}(k)$ has the minimal value of $V_{\mathrm{dyn}}\left(k_{\mathrm{min}}\right)=V_{0}+$ $2\left(4 \pi e^{2} \beta\right)^{1 / 2}-\beta k_{T F}^{2}$ [13-17], and $\rho_{t}$ is then determined from $V_{\mathrm{dyn}}\left(k_{\min }\right)=0$. We note that the first term in Eq. (12) gives the dominant contribution in the determination of the transition density, and including other terms lowers the transition density. Also, although at low temperatures the electron density $\rho_{e}$ can be written as an expansion of the electron chemical potential

$$
\rho_{e} \approx \frac{8 \pi}{3 h^{3}} \mu_{e}^{3}\left[1+\pi^{2}\left(T / \mu_{e}\right)^{2}\right],
$$

which gives an analytical expression for $\partial \mu_{e} / \partial \rho_{e}$, at high temperatures numerical calculations are needed.

The dynamical approach reduces to the so-called thermodynamical approach $[4,12]$ in the long-wavelength limit when the density gradient terms and the Coulomb interaction are neglected $[19,29]$, which leads to the stability condition

$$
V_{\text {ther }}=\frac{\partial \mu_{p}}{\partial \rho_{p}}-\frac{\left(\partial \mu_{n} / \partial \rho_{p}\right)^{2}}{\partial \mu_{n} / \partial \rho_{n}}>0 .
$$

To illustrate the relation between the transition density and the area of the spinodal region, we show in Fig. 3 the instability region of nuclear matter with the boundary determined by $V_{\text {ther }}=0$ and the relative neutron-proton abundance of a hot neutron star in the $\left(\rho_{n}, \rho_{p}\right)$ plane. The cross point, also shown at enlarged scales in the insets, is the transition density from the thermodynamical approach. It is seen that although the neutron-star matter becomes less neutron rich with increasing temperature, the area of the instability region shrinks more quickly with increasing temperature. Furthermore, the stiffness of the symmetry energy also affects the shape and area of the spinodal region. As temperature increases, the spinodal boundaries from different values of $x$ cross with the relative neutron-proton abundance curves at decreasingly small proton densities. For a more detailed discussion on the symmetry energy and temperature effects on the spinodal region, we refer readers to Refs. [30-35]. The following analysis of the transition density and pressure in hot neutron stars is, however, carried out by using the more realistic dynamical approach.

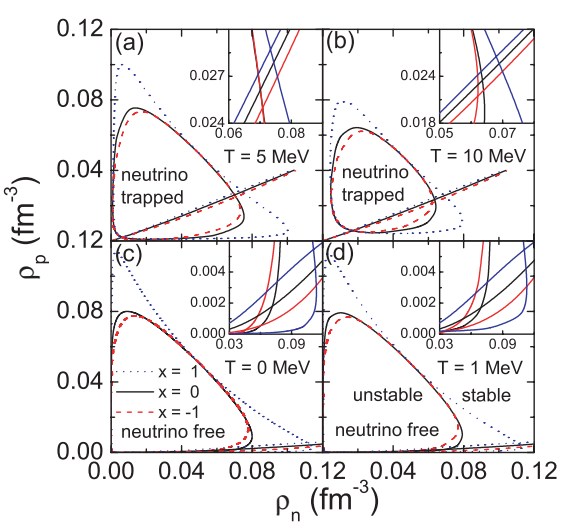

FIG. 3. (Color online) The instability region and the relative neutron-proton abundance of hot neutron-star matter for different temperatures and nuclear symmetry energy parameters. Regions where they cross each other are shown in the insets at enlarged scales.

\section{RESULTS AND DISCUSSIONS}

In this section, we show the temperature dependence of the transition density and pressure in newly born hot neutron stars by using the MDI with different values for the symmetry energy parameter $x$ or the slope parameter $L$ of the symmetry energy.

The dependence of the transition density and pressure in hot neutron stars on the slope parameter $L$ of the symmetry energy at different temperatures is shown in Fig. 4. It is seen that the transition density $\rho_{t}$ generally decreases with increasing value of the slope parameter $L$ of the symmetry energy. As the transition density can be viewed approximately as the beginning of a first-order liquid-gas phase transition, a stiffer symmetry energy, which corresponds to a softer EOS at subsaturation densities, leads thus to a smaller phase transition density and therefore a lower core-crust transition density. Furthermore, the transition density decreases with increasing temperature, and for the neutrino-free matter this is more pronounced for larger values of $L$. The temperature effect can again be understood by the decreasing phase transition density with increasing temperature. For the neutrino-trapped

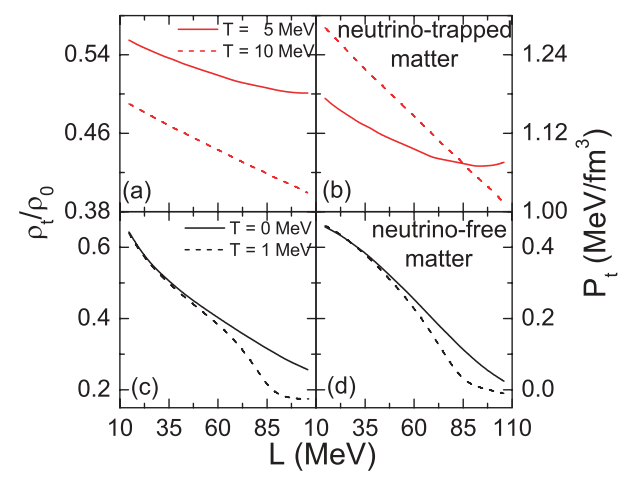

FIG. 4. (Color online) Transition densities $\rho_{t}(\mathrm{a}, \mathrm{c})$ and pressure $P_{t}(\mathrm{~b}, \mathrm{~d})$ as functions of the slope parameter $L$ of the symmetry energy at different temperatures for both the neutrino-trapped matter $(a, b)$ and the neutrino-free matter (c, d). 
matter, the $L$ dependence of the transition density is relatively weak. The weak $L$ dependence is mainly attributable to the fact that the isospin asymmetry is not so large as shown in Fig. 2. We note that a similar temperature dependence of the transition density has been obtained in studies based on Skyrme interactions and relativistic mean-field models [34,36,37]. For the $L$ dependence of the transition density, results from these models are, however, not so clear as different values for the incompressibility $K_{0}$ of the symmetric matter at saturation density and $E_{\text {sym }}\left(\rho_{0}\right)$, which also affect the value of the transition density, have been used.

For the transition pressure $P_{t}$, its value in the neutrinotrapped matter decreases only very slightly with increasing $L$ as a result of the weak $L$ dependence of $\rho_{t}$. Its temperature dependence shows, however, a complicated behavior of slightly higher and smaller values at higher temperatures for smaller and larger values of $L$, respectively. This is attributable to the fact that although the transition density $\rho_{t}$ decreases with increasing temperature, the contribution from leptons increases with increasing temperature. For the neutrino-free matter, $P_{t}$ is seen to decrease rapidly with increasing $L$. As to its temperature dependence, $P_{t}$ in the neutrino-free matter decreases with increasing temperature at larger values of $L$ but shows a weaker temperature dependence for smaller values of $L$. Also, $P_{t}$ is larger for the neutrino-trapped matter than for the neutrino-free matter. Interestingly, $P_{t}$ becomes very small and even negative at $T=1$ with larger $L$ for the neutrino-free matter. This is attributable to the smaller contributions from the leptons and the asymmetric part of the nuclear interactions to the total pressure. Because the pressure at the inner edge of neutron-star crust cannot be negative, our finding thus indicates that either the neutrino-free matter in hot neutron stars cannot reach a temperature above $T=1 \mathrm{MeV}$ or the symmetry energy cannot have a slope parameter larger than $L \sim 100 \mathrm{MeV}$.

The temperature effect on the transition density and pressure for a fixed symmetry energy parameter is demonstrated in Fig. 5 for the symmetry energy parameters $x=1$, $x=0$, and $x=-1$. For the neutrino-trapped matter, the temperature effect is similar for all three $x$ values, and the

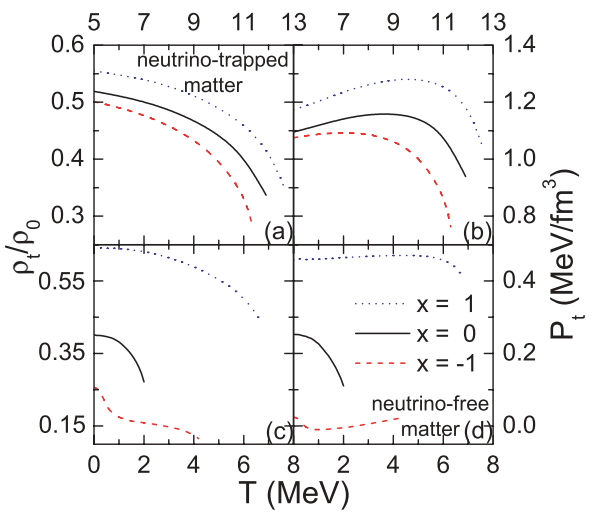

FIG. 5. (Color online) Transition densities $\rho_{t}(\mathrm{a}, \mathrm{c})$ and pressure $P_{t}(\mathrm{~b}, \mathrm{~d})$ as functions of temperature $T$ with $x=0$ and $x=-1$ for both the neutrino-trapped matter $(\mathrm{a}, \mathrm{b})$ and the neutrino-free matter (c, d). Note that different scales for temperatures are used for the neutrino-trapped matter and the neutrino-free matter. transition density $\rho_{t}$ decreases almost linearly with increasing temperature at lower $T$ and decreases quickly at higher $T$. For the neutrino-free matter, although the transition density $\rho_{t}$ decreases smoothly with increasing temperature for $x=1$, it decreases slowly (quickly) at lower temperatures with $x=0$ $(x=-1)$ and quickly (slowly) at higher temperatures. The temperature effects on the transition density reflect those on the spinodal region and the abundance of particle species in the hot neutron-star matter. For the neutrino-trapped matter, the transition pressure $P_{t}$ is seen to be insensitive to the temperature for $x=-1$ but increases slightly with increasing temperature for $x=0$ and $x=1$ at lower $T$, and it decreases with increasing temperature at higher $T$ for all values of $x$. For the neutrino-free matter, it is insensitive to temperature for $x=1$ but decreases with increasing temperature for $x=0$, while for $x=-1$ it drops to a negative value and becomes positive again as the temperature increases. Our results again show that the behavior of $P_{t}$ is dominated by that of $\rho_{t}$ and the contribution from the leptons, with the former decreasing and the latter increasing with increasing temperature.

We have seen in Fig. 3 that as the temperature of the neutron star matter increases, there will be eventually no cross point between the curve of neutron-proton relative abundance and the boundary of the spinodal region, leading to the disappearance of the transition density in hot neutron stars. In such case, the inner crust (nuclear "pasta" phase) disappears and the liquid core expends directly to the outer crust. Determining the critical temperature $T_{c}$ at which the transition density $\rho_{t}$ disappears is thus useful for understanding the structural evolution of newly born hot neutron stars. Figure 6 displays the $L$ dependence of the critical temperature for both the neutrino-trapped matter and the neutrino-free matter. One sees that the critical temperature $T_{c}$ decreases slightly with increasing value of $L$ in the neutrino-trapped matter, but it first decreases and then increases with increasing $L$ in the neutrino-free matter. This complicated behavior is attributable to the isospin and temperature effects on the spinodal region and the relative neutron-proton abundance, as shown in Fig. 3. Our results thus indicate that for neutrino-trapped neutron stars of temperatures higher than $12 \mathrm{MeV}$ or for neutrino-free neutron stars of temperatures higher than $1.5 \mathrm{MeV}$, there exists

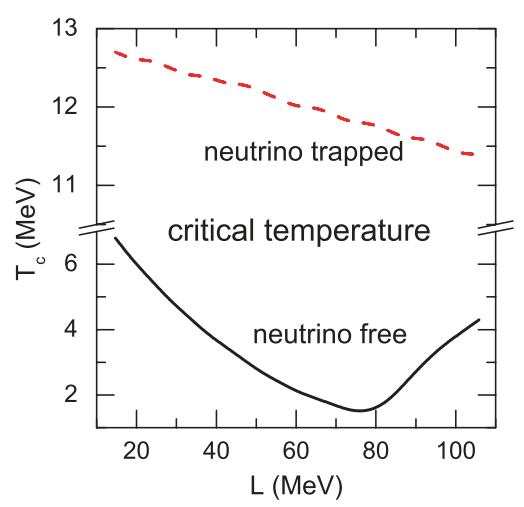

FIG. 6. (Color online) Critical temperature $T_{c}$ as a function of the slope parameters $L$ of the symmetry energy for both the neutrinotrapped matter and the neutrino-free matter. 
no inner crust if the value of $L$ is $70 \sim 80 \mathrm{MeV}$. As newly born hot neutron stars cool, the temperature at which the inner crust can form thus depends on the density dependence of the symmetry energy at subsaturation densities. Again, the magnitude of the critical temperature for both neutrino-trapped and neutrino-free matter is similar to those from Skyrme interactions and relativistic mean-field models [34,37].

The preceding results were obtained using the dynamical approach that includes the effects of both the density gradient terms and the Coulomb interaction. Neglecting these effects, the resulting thermodynamical approach given by Eq. (13) gives higher values for both the transition density and the pressure. This is especially the case for the neutrino-trapped matter and/or for smaller values of $L$ as more electrons are present in such hot neutron stars and the effect owing to the Coulomb interaction becomes more important. Also, there have recently been some studies on the transition density in neutron stars using various nucleon-nucleon interactions $[38,39]$. To see the effect of momentum dependence in the nucleon-nucleon interaction on the transition density and pressure, we have also repeated the preceding calculations using the momentum-independent interaction [40], which gives the same EOS for asymmetric nuclear matter but different single-particle mean-field potential in comparison with the MDI used in the present study, and we find that the momentum-dependent effect on the transition density and pressure in hot neutron stars is small.

\section{SUMMARY}

We have studied the transition density and pressure at the boundary that separates the liquid core from the inner crust of neutron stars using the MDI in both the neutrino-trapped matter and the neutrino-free matter at finite temperatures, which are expected to exist during the early evolution of neutron stars. In particular, we have investigated the effect of nuclear symmetry energy by varying the parameter $x$ in the MDI from 1 to -1 , corresponding to the values $15<L<106 \mathrm{MeV}$ for the slope of nuclear symmetry energy at normal density that were constrained by both the isospin diffusion data [7-9] and other experimental observables [6]. We have found that the transition density and pressure are larger in the neutrinotrapped matter than in the neutrino-free matter. Furthermore, the transition density and pressure are found to roughly decrease with increasing temperature and $L$ for both the neutrino-trapped and the neutrino-free matter, except that the transition pressure shows a complicated relation to the temperature for the neutrino-trapped matter. Also, negative values of the pressure at the transition density have been obtained, which can be used to rule out a very stiff symmetry energy at subsaturation densities. We have also studied the critical temperature above which the inner crust (nuclear "pasta" phase) cannot be formed in newly born neutron stars and found that it depends sensitively on the density dependence of the nuclear symmetry energy at subsaturation densities.

\section{ACKNOWLEDGMENTS}

This work was supported in part by the US National Science Foundation under Grant Nos. PHY-0758115, PHY-0652548, and PHY-0757839, the Welch Foundation under Grant No. A-1358, the Research Corporation under Award No. 7123, the Texas Coordinating Board of Higher Education Award No. 003565-0004-2007, the National Natural Science Foundation of China under Grant Nos. 10675082 and 10975097, MOE of China under project NCET-05-0392, Shanghai Rising-Star Program under Grant No. 06QA14024, the SRF for ROCS, SEM of China, and the National Basic Research Program of China (973 Program) under Contract No. 2007CB815004.
[1] N. Chamel and P. Haensel, Living Rev. Relativ. 11, 10 (2008).

[2] S. B. Ruster, M. Hempel, and J. Schaffner-Bielich, Phys. Rev. C 73, 035804 (2006).

[3] J. M. Lattimer and M. Prakash, Phys. Rep. 333, 121 (2000).

[4] J. M. Lattimer and M. Prakash, Phys. Rep. 442, 109 (2007).

[5] B. A. Li, L. W. Chen, and C. M. Ko, Phys. Rep. 464, 113 (2008).

[6] D. V. Shetty and S. J. Yennello, arXiv:1002.0313v4 [nucl-ex].

[7] M. B. Tsang et al., Phys. Rev. Lett. 92, 062701 (2004).

[8] L. W. Chen, C. M. Ko, and B. A. Li, Phys. Rev. Lett. 94, 032701 (2005); Phys. Rev. C 72, 064309 (2005).

[9] B. A. Li and L. W. Chen, Phys. Rev. C 72, 064611 (2005).

[10] M. B. Tsang, Y. Zhang, P. Danielewicz, M. Famiano, Z. Li, W. G. Lynch, and A. W. Steiner, Phys. Rev. Lett. 102, 122701 (2009).

[11] C. B. Das, S. Das Gupta, C. Gale, and B. A. Li, Phys. Rev. C 67, 034611 (2003).

[12] S. Kubis, Phys. Rev. C 76, 025801 (2007); 70, 065804 (2004).

[13] G. Baym, C. Pethick, and P. Sutherland, Astrophys. J. 170, 299 (1971).

[14] G. Baym, H. A. Bethe, and C. J. Pethick, Nucl. Phys. A 175, 225 (1971).

[15] C. J. Pethick and D. G. Ravenhall, Annu. Rev. Nucl. Part. Sci. 45, 429 (1995).
[16] C. J. Pethick, D. G. Ravenhall, and C. P. Lorenz, Nucl. Phys. A 584, 675 (1995).

[17] K. Oyamatsu and K. Iida, Phys. Rev. C 75, 015801 (2007).

[18] B. Link, R. I. Epstein, and J. M. Lattimer, Phys. Rev. Lett. 83, 3362 (1999).

[19] J. Xu, L. W. Chen, B. A. Li, and H. R. Ma, Phys. Rev. C 79, 035802 (2009); Astrophys. J. 697, 1549 (2009).

[20] A. Burrows and J. M. Lattimer, Astrophys. J. 307, 178 (1986).

[21] H. A. Bethe, Rev. Mod. Phys. 62, 801 (1990).

[22] R. Buras, M. Rampp, H. T. Janka, and K. Kifonidis, Phys. Rev. Lett. 90, 241101 (2003).

[23] H. A. Bethe, G. E. Brown, J. Applegate, and J. M. Lattimer, Nucl. Phys. A 324, 487 (1979).

[24] A. Burrows, Astrophys. J. 334, 891 (1988).

[25] C. J. Horowitz, M. A. Pérez-Garcỉa, and J. Piekarewicz, Phys. Rev. C 69, 045804 (2004).

[26] J. Xu, L. W. Chen, B. A. Li, and H. R. Ma, Phys. Rev. C 75, 014607 (2007).

[27] J. Boguta, Phys. Lett. B 106, 255 (1981).

[28] J. M. Lattimer, C. J. Pethick, M. Prakash, and P. Haensel, Phys. Rev. Lett. 66, 2701 (1991).

[29] J. Margueron and P. Chomaz, Phys. Rev. C 67, 041602(R) (2003). 
[30] B. A. Li, A. T. Sustich, M. Tilley, and B. Zhang, Phys. Rev. C 64, 051303(R) (2001); Nucl. Phys. A 699, 493 (2002).

[31] B. A. Li and C. M. Ko, Nucl. Phys. A 618, 498 (1997).

[32] V. Baran et al., Nucl. Phys. A 632, 287 (1998).

[33] C. Ducoin, Ph. Chomaz, and F. Gulminelli, Nucl. Phys. A 789, 403 (2007).

[34] C. Ducoin, C. Providência, A. M. Santos, L. Brito, and P. Chomaz, Phys. Rev. C 78, 055801 (2008).

[35] S. S. Avancini, L. Brito, D. P. Menezes, and C. Providência, Phys. Rev. C 70, 015203 (2004).
[36] S. S. Avancini, L. Brito, J. R. Marinelli, D. P. Menezes, M. M. W. deMoraes, C. Providência, and A.M. Santos, Phys. Rev. C 79, 035804 (2009).

[37] H. Pais, A. Santos, and C. Providência, Phys. Rev. C 80, 045808 (2009).

[38] A. Sulaksono and Kasmudin, Phys. Rev. C 80, 054317 (2009).

[39] I. Vidaña, C. Providência, A. Polls, and A. Rios, Phys. Rev. C 80, 045806 (2009).

[40] J. Xu, L. W. Chen, B. A. Li, and H. R. Ma, Phys. Lett. B 650, 348 (2007); Phys. Rev. C 77, 014302 (2008). 